\title{
HYPOTHESIS OF QUANTUM AS A DISTRIBUTED SELF-ORGANIZED COMPUTATION BY COLLECTIVE OF PARTICLES
}

\author{
PAVEL V. KURAKIN
}

Received 8 February 2006; Accepted 5 April 2006

Previously we suggested an original interpretation of quantum mechanics. The interpretation supposes that quantum probability amplitudes are a manifestation of self-organization of nature at some deeper level of matter, performed in spatially and temporally distributed manner. Instead of formal description of the concept, the paper shows in detail how it works for basic quantum experiments.

Copyright (c) 2006 Pavel V. Kurakin. This is an open access article distributed under the Creative Commons Attribution License, which permits unrestricted use, distribution, and reproduction in any medium, provided the original work is properly cited.

\section{Introduction}

Quantum mechanics is highly verified in numerous experiments theory of physical phenomena at microscopic level. Still the formalism of quantum theory is very mysterious: the theory formulates not in terms of some directly understood changes of physical fields and particles at each space point during time, like classical field theory does. Instead, a notion of quantum probability amplitude is introduced. Being squared, quantum amplitude (it is a complex number) provides a probability of certain transition from one quantum state of a system (say, a particle) to another one.

The presented interpretation suggests that language of quantum amplitudes can, in principle, be fully consistent with more traditional language of dynamical systems. It is traditionally assumed by pure physicists that such an approach, known as hidden variables theories, is judged to be impossible due to Bell's theorem experiments. In $[5,8]$ we show that assumptions of Bell's theorem do not cover all possible ways to introduce such variables. Instead, Bell's theorem assumes one very special type of hidden variables.

In this paper I pay attention only to detailed description of "how it works" for nonlinear dynamics specialists. Please refer to $[5,8]$ for detailed physical foundation of the suggested approach. 
2 A hidden time hypothesis of quantum

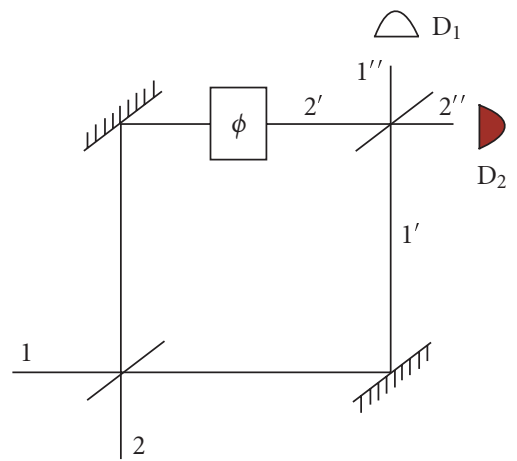

Figure 2.1. Simple 1-photon interference in Mach-Zehnder interferometer.

\section{Simple interference}

Let us assume the most fundamental quantum phenomenon: the interference. Figure 2.1 shows Mach-Zehnder interferometer. Experimenter supplies one photon in direction 1. Standard quantum-mechanical description of this experiment is as follows.

At the 1 st $50 \backslash 50$ beam splitter, quantum state of the photon is split in the superposition of passed and reflected photons:

$$
\left|\psi_{1}\right\rangle \longrightarrow \frac{1}{\sqrt{2}} \cdot\left(\left|\psi_{1^{\prime}}\right\rangle+i \cdot\left|\psi_{2^{\prime}}\right\rangle\right)
$$

Recall that beam splitters rotate the phase of a photon state by $\pi / 2$. Next, each mirror also rotates each state in superposition (multiplies by $i$ ):

$$
\left|\psi_{1^{\prime}}\right\rangle+i \cdot\left|\psi_{2^{\prime}}\right\rangle \longrightarrow i \cdot\left(\left|\psi_{1^{\prime}}\right\rangle+i \cdot\left|\psi_{2^{\prime}}\right\rangle\right) .
$$

Next, path $2^{\prime}$ state is rotated by $\phi$, and before falling onto 2 nd $50 \backslash 50$ beam splitter, the photon's state is

$$
\left|\psi_{1}\right\rangle \longrightarrow \frac{i}{\sqrt{2}} \cdot\left(\left|\psi_{1^{\prime}}\right\rangle+i \cdot \exp (i \cdot \varphi) \cdot\left|\psi_{2^{\prime}}\right\rangle\right)
$$

After passing the 2 nd beam splitter, the state $1^{\prime}$ is split into superposition of states $1^{\prime \prime}$ and $2^{\prime \prime}$ :

$$
\left|\psi_{1^{\prime}}\right\rangle \longrightarrow \frac{1}{\sqrt{2}} \cdot\left(\left|\psi_{1^{\prime \prime}}\right\rangle+i \cdot\left|\psi_{2^{\prime \prime}}\right\rangle\right)
$$

The same does the $2^{\prime}$ state:

$$
\left|\psi_{2^{\prime}}\right\rangle \longrightarrow \frac{1}{\sqrt{2}} \cdot\left(\left|\psi_{2^{\prime \prime}}\right\rangle+i \cdot\left|\psi_{1^{\prime \prime}}\right\rangle\right) .
$$

Summing (2.4) and (2.5) and inserting in (2.3), finally we get

$$
\left|\psi_{1}\right\rangle \longrightarrow \frac{i}{2} \cdot\left[(1-\exp (i \varphi)) \cdot \psi_{1^{\prime \prime}}+i \cdot(1+\exp (i \varphi)) \cdot \psi_{2^{\prime \prime}}\right] .
$$


As a result the probability for detector $\mathrm{D}_{1}$ to detect the photon is as follows:

$$
\left.P_{1}=\left|\frac{1}{2} \cdot(1-\exp (i \varphi)) \cdot\right| \psi_{1^{\prime \prime}}\right\rangle\left.\right|^{2}=\frac{1}{4} \cdot|1-\exp (i \varphi)|^{2}=\sin ^{2}\left(\frac{\varphi}{2}\right)
$$

Accordingly, the probability for detector $\mathrm{D}_{2}$ to detect the photon is $P_{2}=\cos ^{2}(\varphi / 2)$.

If we have no phase shifter at path $2^{\prime}$ at all $(\phi=0)$, we get that detector $\mathrm{D}_{1}$ never clicks. Instead, detector $\mathrm{D}_{2}$ clicks each time.

Here is what hidden time concept provides for this experiment.

(1) At first, scout waves propagate from 1st beam splitter to detectors by all possible paths (Figure 2.2(a)).

Scout waves propagate like standard classical electromagnetic waves. This is why we put two mutually shifted waves at paths $1^{\prime \prime}$ and $2^{\prime \prime}$ : they originate from summing $1^{\prime}$ and $2^{\prime}$. Please note that phase shifter provides some additional rotation of wave phase in the path $2^{\prime}$.

Note also that physical time does not go while these waves "move."

(2) When any detector receives a scout wave, it sends a query wave (Figure 2.2(b)). Query wave can be just reversed in "time" variable scout wave, since Maxwell equations for free electromagnetic field is time-reversible. But in fact only intensity $|\psi|^{2}$ of query waves matters, not the phase. Intensity is a measure of survivability of waves sent by different detectors.

At Figure 2.2(b) a query wave from $\mathrm{D}_{2}$ is stronger (say, $\phi$ in (2.6) and (2.7) is rather small, so $P_{2}>P_{1}$ ), so it has more chances to win a query wave from $\mathrm{D}_{1}$. Still, as one can see from Figure 2.2(c), we admit that nevertheless $D_{1}$ wins. I do so to express that intensity of a query wave means only the probability to win. $\mathrm{D}_{2}$ has, generally speaking, a greater probability to win, but this time $\mathrm{D}_{1}$ wins. If we repeat the experiment many times, the number of each detector's clicks is proportional to its query wave intensity $|\psi|^{2}$. It is exactly what standard quantum mechanics provides.

After winning at the 2 nd beam splitter, query wave of $\mathrm{D}_{1}$ propagates next by all possible ways, that is, by ways provided by scout waves before (Figure 2.2(c)), that is, by $1^{\prime}$ and $2^{\prime}$. Each way's copy of that wave comes to the 1st beam splitter and they also compete there. Since they are of equal intensity, they have equal chances to win the competition. In fact, it is not essential which one wins, because each of these waves is representative of the same single detector $\mathrm{D}_{1}$.

At Figure 2.2(d) path $2^{\prime}$ wins. Green signal means propagation of confirmation wave, which finally comes to detector $D_{1}$. Red signal is refusal wave (just like at Figure 2.2(c)), it propagates up to it's source, that is, 2 nd beam splitter.

The trick here is that all the waves represented above do not propagate in physical time. Only coming of confirmation wave (green) to $\mathrm{D}_{1}$ corresponds to an instant of physical time. Though we have 3 passes of waves from a source to some detector, and eventhough each of these passes obeys Maxwell's equations for free electromagnetic field, finally total time for a photon to travel to the detector is such as if the photon simply moves classically from the source to the detector with light speed. 


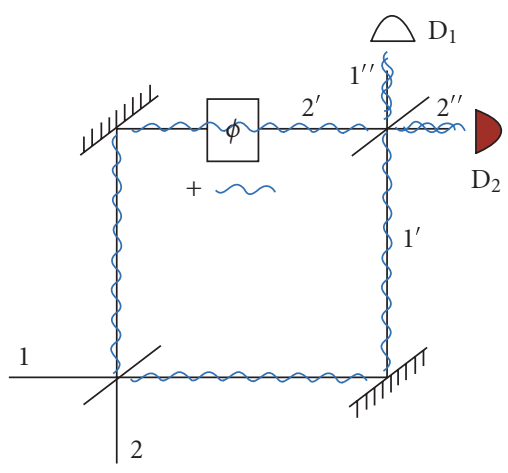

(a)

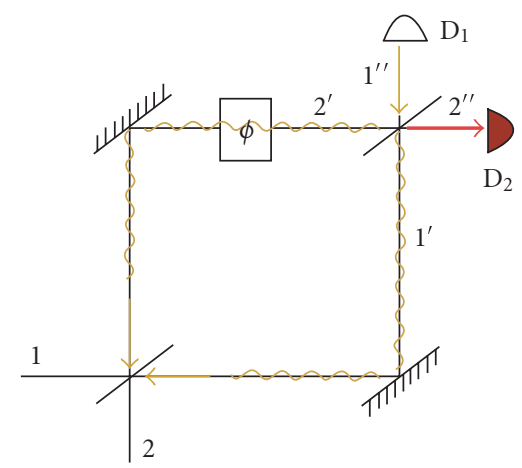

(c)

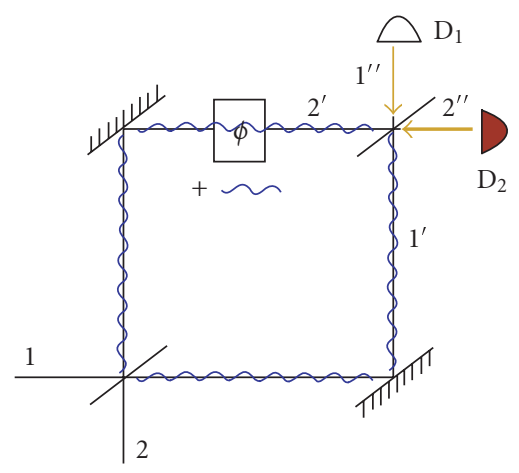

(b)

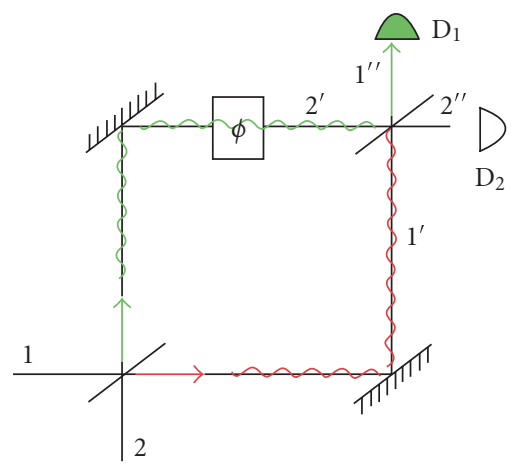

(d)

Figure 2.2. (a) Hidden time at work: scout waves come to each detector by all paths. (b) Hidden time at work: query waves start to compete. (c) Hidden time at work: the winner query wave propagates. (d) Hidden time at work: detector $\mathrm{D}_{1}$ gets the photon.

This sounds very strange, but it is correct if one takes the following four postulates.

(a) Any photon's exchange by charges is accomplished through presented mechanism of 3 passes of waves.

(b) Only final coming of confirmation wave to some detector (a charge) corresponds to an instant of physical time.

(c) Each detector charge serves all scout waves from different source charges it receives consequently; this means that a detector charge sends a query wave in response to some scout wave only after a confirmation (or a refusal) is received for a previously sent query wave.

(d) Detected physical time is simply a number of detected light quanta, properly normalized for each experimental configuration.

One can find more detailed arguments in $[5,8]$. 


\section{Delayed choice}

"Delayed choice" experiment was originally suggested by J. A. Wheeler as a gedankenexperiment. At the moment many versions of delayed choice experiment are proposed and realized. We assume the simplest version as it is presented in [4].

Let us take that we have no phase shifter at the path $2^{\prime}$ of our Mach-Zehnder interferometer at Figure 2.1: $\phi=0$. In this case, $\mathrm{D}_{1}$ never clicks; instead, $\mathrm{D}_{2}$ clicks each time.

Next, suppose that "at the last moment" experimenter decides to perform another experiment than he originally intended: he pulls 2 nd beam splitter out of the photon's path 2 '. "At the last moment" means, of course, before a moment of time, when classically moving photon should reach the beam splitter.

In this case, the photon hits $\mathrm{D}_{1}$ and $\mathrm{D}_{2}$ with equal probabilities. In other words, pulling out 2 nd beam splitter "at the photon's nose" gives the same result as if there were no 2nd beam splitter from the very beginning.

From a standard quantum mechanical viewpoint, the explanation is as follows. We take into account all classically possible paths in space-time for the photon; each path provides its own complex-valued amplitude $\psi_{i}$. Total amplitude of considered transition is the sum $\sum \psi_{i}$ for all paths.

So, when the 2nd (upper part at Figure 2.1) beam splitter is removed from the interferometer at a proper time, each classically possible path in space-time does not encounter that beam splitter on its way. Thus destructive interference for $\mathrm{D}_{1}$ disappears.

What explanation will hidden time approach provide for delayed choice experiment? First, let us take for simplicity that 2nd beam splitter is not removed indeed from its position; instead it is in some way switched off to be transparent for light. Switching off is accomplished by some electromagnetic signal. Again, we can take for simplicity that this signal consists of a single quantum of light.

Recall that according to rule (a) (Section 4) this quantum propagates obeying the same general mechanism we outlined before. This means that initially a scout wave of this quantum arrives to the beam splitter (Figure 3.1(a)). We assume that we switch off the beam splitter "at the nose" of the photon which propagates within the interferometer. This means that scout wave of switching photon arrives (in hidden time!) to the beam splitter earlier than the scout wave of interferometer photon.

According to rule (c) from Section 4, this means that the beam splitter (we take it as if it is a single charge) serves switching photon first. Interferometer's photon scout waves stand unserved (and with no further propagation) until switching photon is ultimately served: that is, until it is absorbed or rejected by the beam splitter.

So, the beam splitter sends query wave in response to switching photon's scout wave (Figure 3.1(b)). Then, it receives confirmation wave, that is, the switching photon itself (Figure 3.1(c)). Of course, we suggest that the beam splitter receives confirmation rather than refusal, since we assume that we do switch off the beam splitter!

Only after switching off the beam splitter (which is equivalent to receiving confirmation wave in hidden time) scout waves of interferometer's photon propagate next from the beam splitter to detectors. And now they do not interfere (Figure 3.1(d)), since the beam splitter is practically absent! 


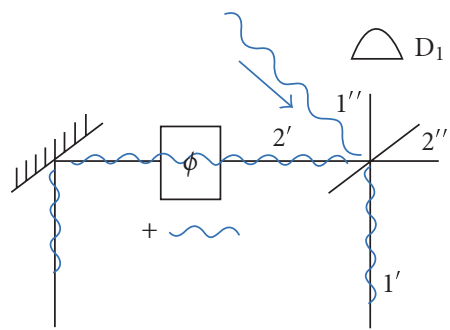

(a)

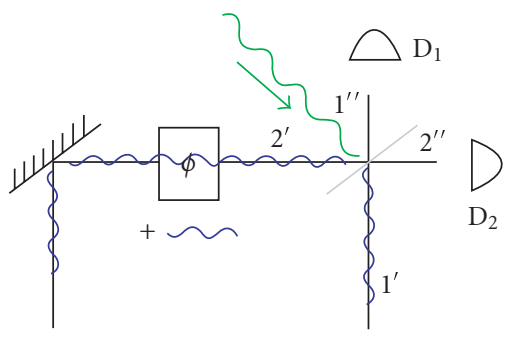

(c)

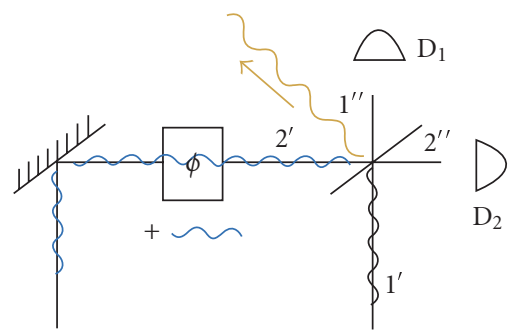

(b)

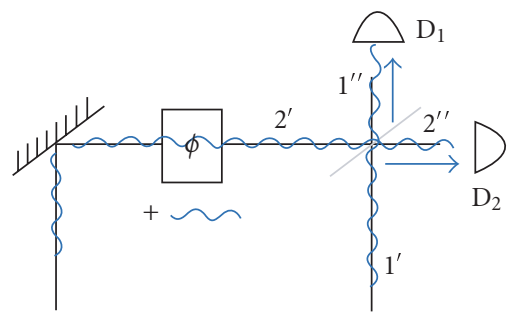

(d)

Figure 3.1. (a) Hidden time at work: scout wave of control photon comes to 2 nd beam splitter. (b) Hidden time at work: query wave of control photon comes from 2 nd beam splitter. (c) Hidden time at work: confirmation wave of control photon comes to 2 nd beam splitter. (d) Hidden time at work: scout waves do not interfere since beam splitter is transparent.

So in this case query waves from $\mathrm{D}_{1}$ and $\mathrm{D}_{2}$ compete to each other at the 1 st beam splitter, like at Figure 2.2(d), and finally any detector can click.

\section{Entanglement: the core}

Again, we start from what standard quantum theory provides for entanglement. In quantum optics domain we can get entangled photons most simply by $50 \backslash 50$ beam splitter (Figure 4.1). Let us take that we send horizontally polarized photon to input 1 and vertically polarized photon to input 2 of the beam splitter. Since the $50 \backslash 50$ beam splitter adds a phase factor $i$ to redirected "half" of each photon, we have at the outputs

$$
\begin{aligned}
& |H\rangle_{1}|V\rangle_{2} \longrightarrow \frac{1}{2} \cdot\left(|H\rangle_{1^{\prime}}+i \cdot|H\rangle_{2^{\prime}}\right) \cdot\left(|V\rangle_{2^{\prime}}+i \cdot|V\rangle_{1^{\prime}}\right) \\
& \quad=\frac{1}{2} \cdot\left(|H\rangle_{1^{\prime}}|V\rangle_{2^{\prime}}-|H\rangle_{2^{\prime}}|V\rangle_{1^{\prime}}+i \cdot\left(|H\rangle_{1^{\prime}}|V\rangle_{1^{\prime}}+|H\rangle_{2^{\prime}}|V\rangle_{2^{\prime}}\right)\right)
\end{aligned}
$$

Under the condition of detecting a single photon per each detector, this state is finally projected onto

$$
|H\rangle_{1}|V\rangle_{2} \longrightarrow \frac{1}{2} \cdot\left(|H\rangle_{1^{\prime}}|V\rangle_{2^{\prime}}-|H\rangle_{2^{\prime}}|V\rangle_{1^{\prime}}\right)
$$




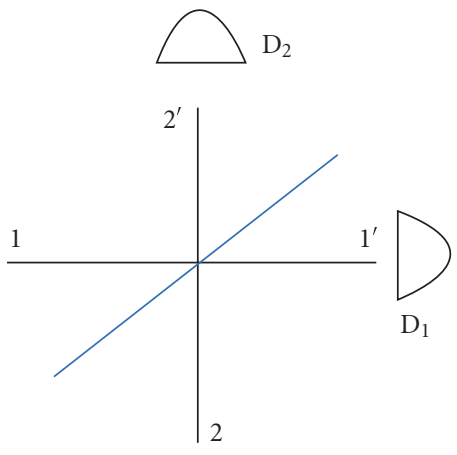

Figure 4.1. $50 \backslash 50$ beam splitter produces entanglement of two photons.

This state is "maximally entangled": detecting $|H\rangle$ in one detector inevitably leads to detecting $|V\rangle$ in the other, and otherwise.

On the other side, imagine that we use not $|H\rangle$ and $|V\rangle$ polarizations for input photons; instead, we use arbitrary $\left|P_{1}\right\rangle$ and $\left|P_{2}\right\rangle$-the derivation of final result does not depend on this substitution. Next, let us take that $P_{1}=P_{2}=P$, that is, our photons are indistinguishable. In that case, (4.1) reduces to

$$
|P\rangle_{1}|P\rangle_{2} \longrightarrow \frac{i}{2} \cdot\left(|P\rangle_{1^{\prime}}|P\rangle_{1^{\prime}}-|P\rangle_{2^{\prime}}|P\rangle_{2^{\prime}}\right) .
$$

State (4.3) means that each time only one detector clicks or, in other words, the photons always go together and never separately! It is Hong-Ou-Mandel effect.

Here is what hidden time concept can offer to explain entanglement. At the inputs of $50 \backslash 50$ beam splitter we have $|H\rangle$ scout wave at input 1 and $|V\rangle$ wave at input 2 (Figure 4.2(a)).

Recall that the beam splitter splits each wave:

$$
\left|\psi_{1}\right\rangle \longrightarrow \frac{1}{\sqrt{2}} \cdot\left(\left|\psi_{1^{\prime}}\right\rangle+i \cdot\left|\psi_{2^{\prime}}\right\rangle\right)
$$

Recall also that in hidden time model each term of this sum represents some new thread of a scout wave. When we multiply such sums, from hidden time view, this means that each term thread in each sum becomes "tied" or "married" to each term thread in the other sum. So, each term in each factor is split again, while the factor of splitting is the number of terms in a paired factor.

Thus after leaving the beam splitter we have $2 \times 2=4$ groups of tied (or married) scout waves (Figure 4.2(b)):

$$
\begin{array}{ll}
S_{\text {out }}^{1}=\left|H_{1^{\prime}}\right\rangle\left|V_{2^{\prime}}\right\rangle, & S_{\text {out }}^{2}=\left|H_{1^{\prime}}\right\rangle\left|V_{1^{\prime}}\right\rangle, \\
S_{\text {out }}^{3}=\left|H_{2^{\prime}}\right\rangle\left|V_{1^{\prime}}\right\rangle, & S_{\text {out }}^{1}=\left|H_{2^{\prime}}\right\rangle\left|V_{2^{\prime}}\right\rangle .
\end{array}
$$


8 A hidden time hypothesis of quantum

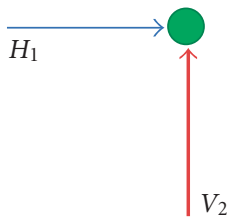

$$
S_{\text {in }}=\left|H_{1}\right\rangle\left|V_{2}\right\rangle
$$

(a)

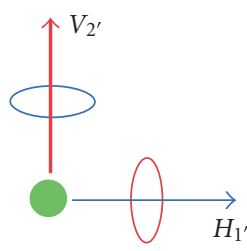

$S_{\text {out }}^{1}=\left|H_{1^{\prime}}\right\rangle\left|V_{2^{\prime}}\right\rangle$

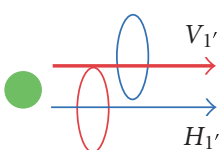

$S_{\text {out }}^{2}=\left|H_{1^{\prime}}\right\rangle\left|V_{1^{\prime}}\right\rangle$

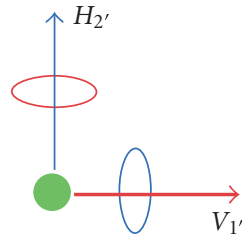

$S_{\text {out }}^{3}=\left|H_{2^{\prime}}\right\rangle\left|V_{1^{\prime}}\right\rangle$

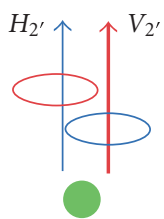

$S_{\text {out }}^{4}=\left|H_{2^{\prime}}\right\rangle\left|V_{2^{\prime}}\right\rangle$

(b)

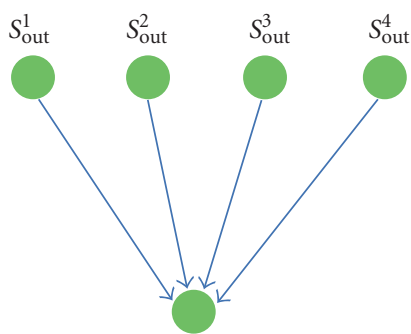

$B S$

(c)

Figure 4.2. (a) Entanglement: the input state. (b) Entanglement: 4 bunched output states. (c) Output states compete as independent units.

Let us refer to each group of tied threads as a bunch. Each of these bunches of scout waves correspond (with appropriate factors) to some term in (4.1) (when in expanded view). Each bunch has a kind of connection knot, where single-photon waves are "tied." Any scout wave within bunch can be viewed as to be "married" with its partner. We mark it by a kind of "marriage rings" at Figure 4.2(b).

When we say "the second photon" we mean that it is in some way distinguishable from the first photon. Turn back to a series of Figure 2.2. The second beam splitter of the MachZehnder interferometer does not make additional splitting of daughter scout waves, since they are indistinguishable!

In standard quantum mechanical formalism we add amplitudes for indistinguishable events and multiply them for distinguishable (independent) events. The same we do for scout wave threads in hidden time theory! 
Next, imagine that each one-photon scout wave in each bunch behaves just the same way we described above: it travels to appropriate detector and is (possibly) summed there with identical copies of itself (if any) (that should be copies within the same bunched state! In other words, each one-photon scout wave within a bunched thread can divide and converge along its way like in 1-photon Mach-Zehnder interferometer), like it takes place in simple interference. Then that detector sends a query wave with intensity equal to squared sum of received scout waves.

Previously we suggested that the phase is inessential for a query wave. Let us now assume that it is not so: a query wave has phase variable, but it is constant along the way back from appropriate detector, and equal to the phase of scout wave at this detector. So each (one-photon) query wave provides a standard (one-photon!) quantum mechanical amplitude for a particular detector.

When any (one-photon) query wave comes from its detector to bunch connection knot (green circle in Figure 4.2(b)) it meets its partner within the bunch. Amplitudes of both waves are multiplied as complex numbers. So we get each of the values of $S_{\text {out }}^{1}, S_{\text {out }}^{2}, S_{\text {out }}^{3}$, $S_{\text {out }}^{4}$ to be physically calculated in hidden time.

The following is quite trivial: bunched states compete to each other just like we discussed above for ordinary query waves in one-photon experiment (Figure 4.2(c)). This competition leads for some single bunched state to win, and each detector gets its photon (if any) for this winner state.

\section{Entanglement: change of basis}

We want to argue that hidden time model of entanglement does not simply look like true quantum entanglement, but is exactly equivalent to what quantum mechanics provides. Let us have an entangled two-component system:

$$
\Psi_{A B}=\frac{1}{\sqrt{2}} \cdot\left(\left|0_{A}\right\rangle\left|1_{B}\right\rangle-\left|1_{A}\right\rangle\left|0_{B}\right\rangle\right)
$$

Let us change observation basis in the following way:

$$
\begin{aligned}
\left|X_{A, B}\right\rangle & =\frac{1}{\sqrt{2}}\left(\left|0_{A, B}\right\rangle+\left|1_{A, B}\right\rangle\right), \\
\left|Y_{A, B}\right\rangle_{A, B} & =\frac{1}{\sqrt{2}}\left(\left|0_{A, B}\right\rangle-\left|1_{A, B}\right\rangle\right) .
\end{aligned}
$$

If $|0\rangle$ and $|1\rangle$ denote polarization states, then such change of basis means that we rotate optical axes of crystals we use by $\pi / 2$ counterclockwise. From these equations we find that

$$
\begin{aligned}
& \left|0_{A, B}\right\rangle=\frac{1}{\sqrt{2}}\left(\left|X_{A, B}\right\rangle+\left|Y_{A, B}\right\rangle\right), \\
& \left|1_{A, B}\right\rangle=\frac{1}{\sqrt{2}}\left(\left|X_{A, B}\right\rangle-\left|Y_{A, B}\right\rangle\right) .
\end{aligned}
$$




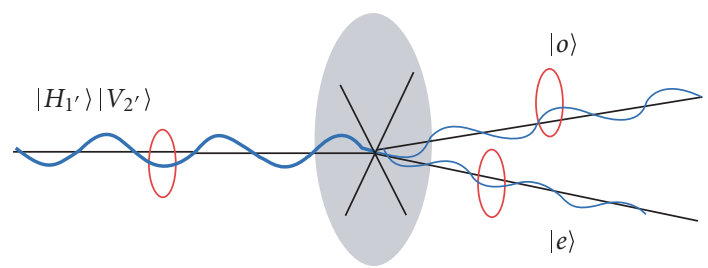

Figure 5.1. Married $\left|H_{1^{\prime}}\right\rangle$ scout wave is split by birefringent crystal into married $|o\rangle$ and $|e\rangle$ scout waves, each of half intensity.

If we substitute these expressions into (5.1), we get

$$
\begin{aligned}
& \Psi_{A, B}= \frac{1}{2 \sqrt{2}}\left[\left(\left|X_{A}\right\rangle+\left|Y_{A}\right\rangle\right)\left(\left|X_{B}\right\rangle-\left|Y_{B}\right\rangle\right)-\left(\left|X_{A}\right\rangle-\left|Y_{A}\right\rangle\right)\left(\left|X_{B}\right\rangle+\left|Y_{B}\right\rangle\right)\right] \\
&=\frac{1}{2 \sqrt{2}}\left[\left|X_{A}\right\rangle\left|X_{B}\right\rangle-\left|X_{A}\right\rangle\left|Y_{B}\right\rangle+\left|Y_{A}\right\rangle\left|X_{B}\right\rangle-\left|Y_{A}\right\rangle\left|Y_{B}\right\rangle\right. \\
&\left.\quad-\left|X_{A}\right\rangle\left|X_{B}\right\rangle-\left|X_{A}\right\rangle\left|Y_{B}\right\rangle+\left|Y_{A}\right\rangle\left|X_{B}\right\rangle+\left|Y_{A}\right\rangle\left|Y_{B}\right\rangle\right] \\
&=-\frac{1}{\sqrt{2}}\left(\left|X_{A}\right\rangle\left|Y_{B}\right\rangle-\left|Y_{A}\right\rangle\left|X_{B}\right\rangle\right) .
\end{aligned}
$$

So in $(X, Y)$ basis we get the same entangled state (change of overall phase by $\pi$ has no physical sense) as in $(0,1)$ basis. It is crucial for us here whether hidden time model is able to reproduce this feature of standard quantum theory.

We suggest that such a change of basis makes no difficulties for hidden time approach. Since at the input and output of a beam splitter we have $|0\rangle$ and $|1\rangle$ ket states only, we have no changes of hidden time signals until they reach optical devices (birefringent crystals). In other words, in hidden time we have $|H\rangle|V\rangle$ single-photon waves and the same bunched waves for two photons as before:

$$
\begin{array}{ll}
S_{\text {out }}^{1}=\left|H_{1^{\prime}}\right\rangle\left|V_{2^{\prime}}\right\rangle, & S_{\text {out }}^{2}=\left|H_{1^{\prime}}\right\rangle\left|V_{1^{\prime}}\right\rangle, \\
S_{\text {out }}^{3}=\left|H_{2^{\prime}}\right\rangle\left|V_{1^{\prime}}\right\rangle, & S_{\text {out }}^{1}=\left|H_{2^{\prime}}\right\rangle\left|V_{2^{\prime}}\right\rangle .
\end{array}
$$

Next, if we rotate the crystal located at the path $1^{\prime}$, it is exactly this location, after which $\left|H_{1^{\prime}}\right\rangle$ or $\left|V_{1^{\prime}}\right\rangle$ scout wave (alone or within a bunch) is split into sum of ordinary $|o\rangle$ and extraordinary $|e\rangle$ waves-Figure 5.1.

Like before, $|o\rangle$ and $|e\rangle$ scout waves propagate to detectors, then come back to splitting point, that is, the birefringent crystal we use, and compete there. Next, regardless of which of two branches $|o\rangle$ and $|e\rangle$ wins, initial scout wave $\left.S_{\text {out }}^{1}=\| H_{1^{\prime}}\right\rangle\left|V_{2^{\prime}}\right\rangle$ propagates backward with its own intensity and phase to the beam splitter we use to compete there with other bunched (married) states we have in the system.

In other words, from a hidden time viewpoint, change of basis at detection location does not affect competition of initial scout waves. 
So, within hidden time approach, entanglement is indeed independent of change of basis.

\section{Entanglement: delayed choice}

From previous description it should be clear that using time-varying analyzers in entanglement experiments, like in classical experimental work [2], brings no new challenge to hidden time approach.

The core idea is very simple:

(1) first, scout waves find all detectors and "frozen" physical states of all devices involved, the very time is "frozen;"

(2) second, scout waves come back to their sources and compete there;

(3) third, winner waves return to appropriate detectors and "unfreeze the time."

This general scheme just ignores our naive tricks with fast switching of analyzers. We think we are fast to switch the devices we use, but hidden time does not think so.

\section{Conclusions}

Suggested hypothesis views basic quantum phenomena as a manifestation of selforganized computations of collectives of particles, performed in spatially and temporally distributed manner. Some possible consequences for physical theory and applications are discussed in [5-8]. Other consequences are planned for further papers.

It is notable that the idea of back propagation was formulated many times to interpret quantum mechanics $[1,3]$. Still, the idea of hidden time, which is a trivial and obvious next step, has not been yet formulated.

\section{References}

[1] Y. Aharonov and L. Vaidman, The Two-State Vector Formalism of Qauntum Mechanics, http:// arxiv.org/abs/quant-ph/0105101.

[2] A. Aspect, J. Dalibard, and G. Roger, Experimental test of Bell's inequalities using time-varying analyzers, Physical Review Letters 49 (1982), no. 25, 1804-1807.

[3] J. G. Cramer, The transactional interpretation of quantum mechanics, Reviews of Modern Physics 58 (1986), no. 3, 647-687.

[4] A. C. Elitzur, S. Dolev, and A. Zeilinger, Time-Reversed EPR and the Choice of Histories in Quantum Mechanics, http://arxiv.org/abs/quant-ph/0205182.

[5] P. V. Kurakin, Hidden variables and hidden time in quantum theory, http://arxiv.org/abs/ quant-ph/0504089.

[6] P. V. Kurakin and G. G. Malinetskii, Toy quantum mechanics using hidden variables, Discrete Dynamics in Nature and Society 2004 (2004), no. 2, 357-361.

[7] Pavel V. Kurakin and George G. Malinetskii, A Simple Hypothesis on the Origin and Physical Nature of Quantum Superposition of States, http://arxiv.org/abs/physics/0505120.

[8] P. V. Kurakin, G. G. Malinetskii, and H. Bloom, Conversational (dialogue) model of quantum transitions, http://arxiv.org/abs/quant-ph/0504088.

Pavel V. Kurakin: Keldysh Institute of Applied Mathematics, Russian Academy of Sciences, 125047 Moscow, Russia

E-mail address: kurakin.pavel@gmail.com 


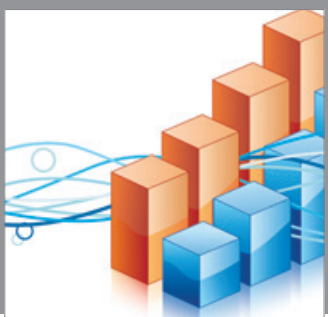

Advances in

Operations Research

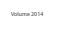

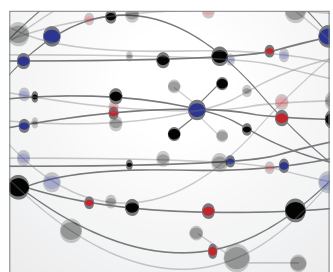

\section{The Scientific} World Journal
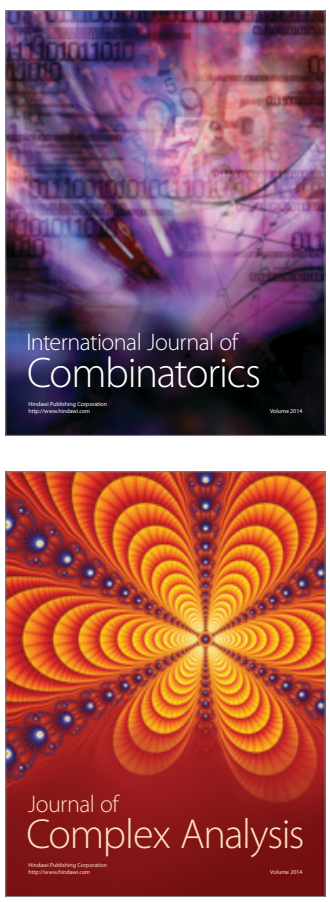

International Journal of

Mathematics and

Mathematical

Sciences
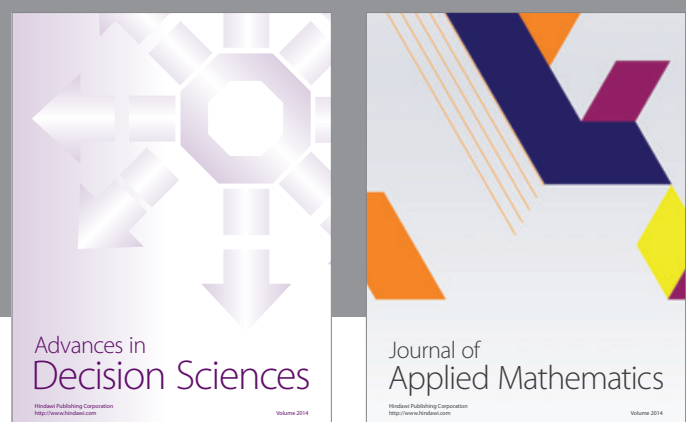

Journal of

Applied Mathematics
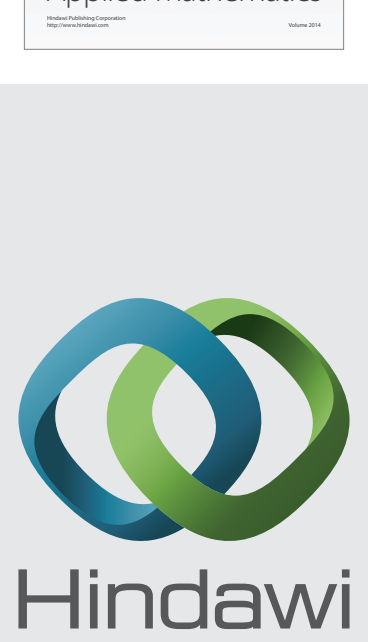

Submit your manuscripts at http://www.hindawi.com
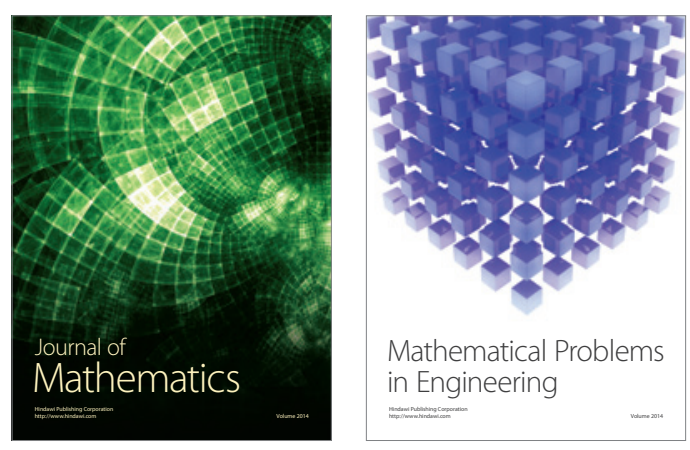

Mathematical Problems in Engineering
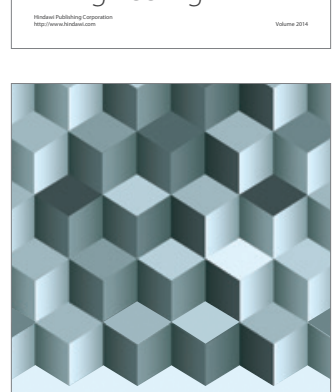

Journal of

Function Spaces
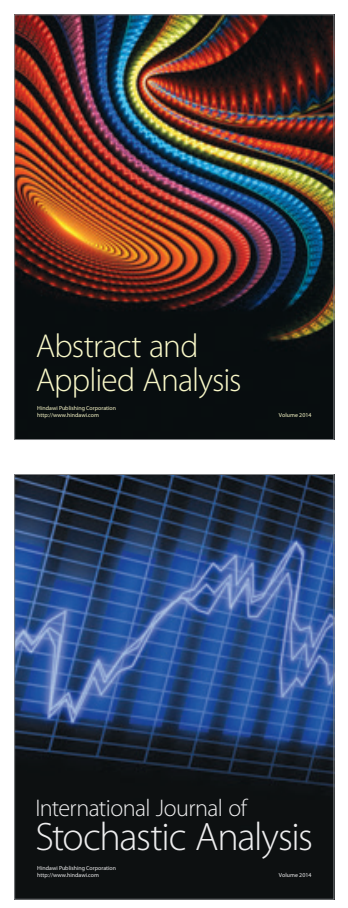

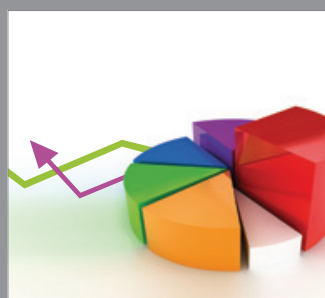

ournal of

Probability and Statistics

Promensencen
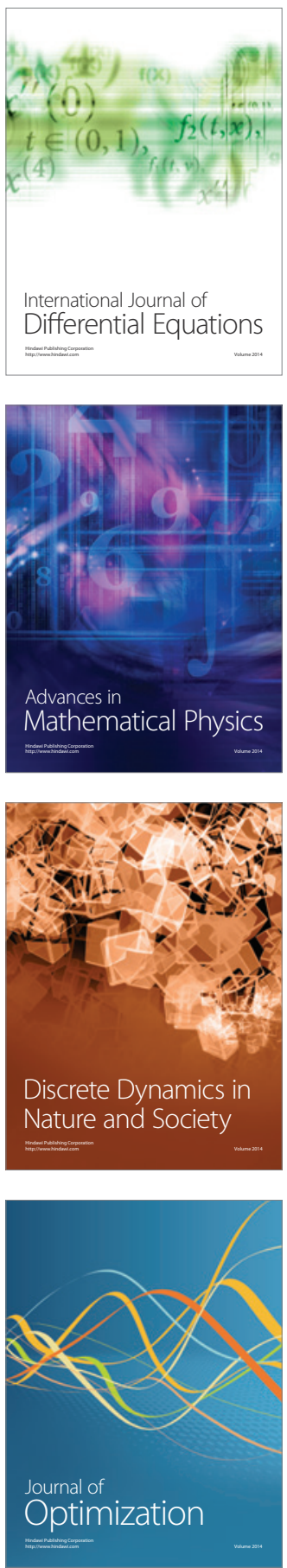